\title{
Ensamblando el embrión de la biología del desarrollo en Uruguay
}

\section{Building the embryo of developmental biology in Uruguay}

\author{
FLAVIO R. ZOLESSI \\ Facultad de Ciencias, Universidad de la República, Uruguay \\ Institut Pasteur de Montevideo, Uruguay. \\ fzolessi@fcien.edu.uy \\ ORCID: 0000-0003-2405-994X \\ NIBIA BEROIS \\ Facultad de Ciencias, Universidad de la República, Uruguay \\ berois@gmail.com \\ ORCID: 0000-0002-6082-5966 \\ M. MÓNICA BRAUER \\ Instituto de Investigaciones Biológicas Clemente Estable, Uruguay \\ brauer2009@gmail.com \\ ORCID: 0000-0003-4616-3625

\section{ESTELA CASTILLO} \\ Facultad de Ciencias, Universidad de la República, Uruguay. \\ castillo@fcien.edu.uy \\ ORCID: 0000-0002-6053-7585
}

Zolessi FR, Berois N, Brauer MM, Castillo E (2021) Building the embryo of developmental biology in Uruguay. International Journal of Developmental Biology, Vol. 65 Nos. 1/2/3 (2021) pp. 71-76 of the Special Issue Developmental Biology in IberoAmerica - Part 1. https://doi.org/10.1387/ijdb.200141fz

\section{Resumen}

En Uruguay, un país con una población pequeña y, por tanto, una pequeña comunidad científica, no existieron embriólogos clásicos como tales en el pasado. A partir de la década de 1950, sin embargo, un cúmulo de condiciones favorables dio lugar a grupos de investigación muy activos y modernos en los campos de la citología y la fisiología, que eventualmente contribuyeron a la biología del desarroIlo. El advenimiento de una larga dictadura entre los años setenta y ochenta provocó dos cosas: un fuerte rezago en la investigación local y la migración de jóvenes investigadores que aprendieron en el extranjero nuevas disciplinas y tecnologías. El retorno a la democracia permitió el regreso de algunos, ahora como investigadores sólidos, y junto con los que se quedaron, construyeron un programa de formación de posgrado previamente inexistente y una academia globalmente integrada que fomentó la diversidad de disciplinas de investigación, incluida la biología del desarrollo. En el presente trabajo destacamos el aporte clave de investigadores pioneros y el importante papel que jugaron las instituciones académicas y financiadoras nacionales en el crecimiento y consolidación de la biología del desarrollo en nuestro país.

Palabras clave: historia de la ciencia; ciencia en Latinoamérica; citología; embriología.

\section{Abstract}

In Uruguay, a country with a small population, and hence a small scientific community, there were no classical embryologists as such in the past. From 
the decade of 1950, however, a cumulus of favorable conditions gave rise to highly active and modern research groups in the fields of cytology and physiology, that eventually contributed to developmental biology. The advent of a long dictatorship between de 1970's and 1980's caused two things: a strong lag in local research and the migration of young investigators who learned abroad new disciplines and technologies. The coming back to democracy allowed for the return of some, now as solid researchers, and together with those who stayed, built a previously inexistent postgraduate training program and a globally-integrated academy that fostered diversity of research disciplines, including developmental biology. In the present work we highlight the key contribution of pioneer researchers and the significant role played by academic and funding national institutions in the growth and consolidation of developmental biology in our country.

Keywords: history of science; science in Latin America; cytology; embryology

\section{Antecedentes históricos: el huevo}

Como disciplina, se suele asumir que la biología del desarrollo es el derivado natural de la embriología clásica, tras el advenimiento de la biología molecular. Esta idea es bastante aplicable a los países desarrollados, donde toda la gama de temas biológicos se investigaba activamente alrededor de la década de 1970 . Pero, ¿qué pasa con países con poca población y un presupuesto muy bajo dedicado a la ciencia? Varias disciplinas estarían sub-representadas y serían eventualmente inexistentes, a menos que investigadores de diversas áreas decidieran incursionar en temas inexplorados. Este parece ser el caso de la embriología en Uruguay. Además del trabajo de algunos naturalistas, la mayor parte de la investigación en biología de principios y mediados del siglo XX en Uruguay se centró en la investigación biomédica, en gran parte dirigida inicialmente por la Facultad de Medicina (Universidad de la República) y más tarde también por el instituto de investigación fundado por Clemente Estable (1894-1976), y que ahora lleva su nombre (Instituto de Investigaciones Biológicas Clemente Estable, IIBCE). La investigación en anatomía y fisiología estaba muy sesgada hacia la investigación del sistema nervioso, lo cual muy probablemente se debía a la formación temprana de Estable en el laboratorio del Premio Nobel Santiago Ramón y
Cajal en Madrid en la década de 1920. Por otro lado, Estable tuvo un gran socio en Francisco Alberto Sáez, quien fue el responsable del establecimiento de una importante escuela de citogenética en Uruguay y la región, influyendo también en algunos de los trabajos que describimos a continuación (Fig.1A; para relatos generales de estos primeros años, consulte Drets, 2013; Pellegrino et al., 2017).

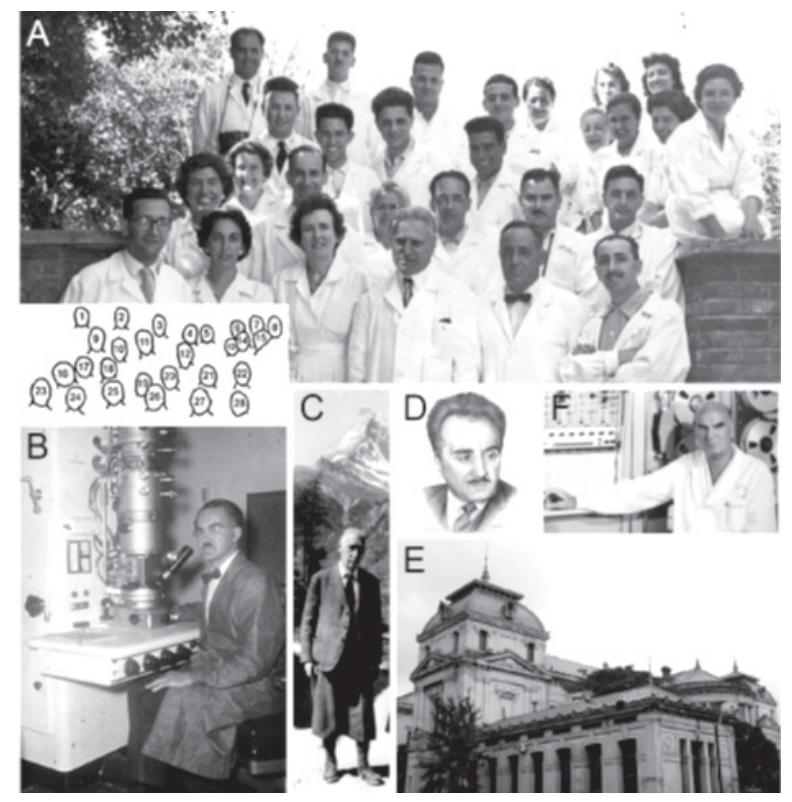

Figura 1. Los inicios. (A) Parte del personal científico del IIBCE ca. 1956: 1, Raúl Vaz Ferreira; 2, Oscar Vincent; 3, Eduardo Pagani; 4, Álvaro Díaz Maynard; 5, Aída Pintos; 6, Amalia Laguardia; 7, Margarita Pérez; 8, Nadir Brum; 9, José Sas; 10, Omar Trujillo-Cenóz; 11, Br. Amatti; 12, Aníbal Alvarez; 13, Elsa Trinkle; 14, Gloria Martínez; 15, Adela Wittemberger; 16, Mercedes Rovira; 17, Nilsa Iriondo; 18, Hugo Freire; 19, Tomasa Rodríguez; 20, José María Martínez; 21, Walter Acosta Ferreira; 22, Héctor Franco Raffo; 23, Máximo E. Drets; 24, Nubia F. de San Germán; 25, María Isabel Ardao; 26, Clemente Estable; 27, Francisco Alberto Sáez; 28, J. Roberto Sotelo. (B) Eduardo De Robertis, frente a un microscopio electrónico. (C) Giuseppe Levi, practicando montañismo. (D) Washington Buño. (E) Edificio de la Facultad de Medicina de Montevideo, visto desde la esquina noreste donde, desde el primer piso hacia arriba, todavía se ubica el Departamento de Histología y Embriología. El Laboratorio de Cultivo de Tejidos, liderado primero por Horacio Goyena y luego por Cristina Arruti, ocupó la cima de la torre. (F) Roberto Caldeyro Barcia en el Departamento de Electrónica. A, archivo IIBCE, clave y nombres tomados de Drets, 2013; B, de Campos Muñoz, 2013; C, de www.nico.ottolenghi.unito.it; $D$, dibujo de M. Picón, tomado de la oficina del Decanato de la Facultad de Medicina, Universidad de la República; F, de Beretta Curi, 2006. 
Los inicios: del embrión a una larva en rápido crecimiento

No es sorprendente que la investigación en neurociencias en Uruguay se haya realizado principalmente a nivel celular, con un gran desarrollo de la citología y la electrofisiología. En la década de 1950, el estudio estructural de las células exigió el uso de la microscopía electrónica, y este es uno de los desarrollos técnicos que sin duda condujeron a algunas de las contribuciones iniciales más importantes de nuestro país a las disciplinas prospectivas de "biología celular" y "biología del desarrollo". En 1950, el instituto liderado por Estable se trasladó a un nuevo edificio, donde todavía se encuentra hoy y, con el apoyo de la Fundación Rockefeller, adquirió el primer microscopio electrónico de transmisión de América Latina. Estable invitó a Eduardo De Robertis (1913-1988; reconocido investigador argentino que entonces vivía en Estados Unidos) para dirigir el recién creado Departamento de Ultraestructura Celular, que nucleó a varios investigadores y fue considerado como "la primera escuela latinoamericana de microscopía electrónica" (Fig. 1B). De Robertis estaba interesado en comprender la estructura fina de las neuronas de los vertebrados, siendo el responsable del descubrimiento de los microtúbulos en los axones y de las vesículas sinápticas. Parte de su trabajo en Uruguay incluyó descripciones exquisitas de la formación del segmento externo de los fotorreceptores de ratón, desde el cilio inicial hasta el desarrollo de los discos membranosos (De Robertis, 1956), un trabajo que continuó cuando regresó a Buenos Aires. Como nota al margen, el hijo de De Robertis, Edward M. (1947), aunque nació en los Estados Unidos, creció y se graduó con honores como médico en Uruguay, antes de continuar su carrera de investigador en el extranjero, para convertirse en un biólogo del desarrollo reconocido mundialmente y uno de los líderes en el campo de la inducción neuronal.

Otras contribuciones relevantes de este laboratorio a la biología del desarrollo, son los estudios ultraestructurales pioneros sobre complejos sinaptonémicos de células meióticas realizados por J. Roberto Sotelo (1915-1985), Rodolfo Wettstein (1942-2009) y Omar Trujillo-Cenóz (1933) (Fig. 1A). Como ejemplo, e inicialmente como una colaboración con uno de los fundadores de la microscopía electrónica aplicada a la biología, Keith R. Porter (1912-1997), Sotelo inició una caracterización ultraestructural de ovocitos de mamíferos. Esta investigación pronto llevó al primer reporte de la presencia de complejos sinaptonémicos en estas células (Sotelo, 1959; Sotelo y Trujillo-Cenóz, 1960), solo descritos previamente en espermatocitos.
En estudios posteriores, utilizando micrografías de alrededor de un centenar de secciones ultrafinas seriadas, Sotelo y Wettstein fueron capaces de realizar reconstrucciones en $3 \mathrm{D}$ de todo el núcleo meiótico, así como seguir la trayectoria completa de cada bivalente desde su inserción en la membrana nuclear (Sotelo y Wettstein, 1964; Wettstein y Sotelo, 1967). Este enfoque confirmó el concepto de que los complejos sinaptonémicos representan cromosomas homólogos apareados, y permitió predecir su papel clave en la recombinación meiótica. En conjunto, estos estudios dieron lugar a una nueva disciplina: la citogenética ultraestructural. El trabajo de biología celular de la meiosis iniciado por Sotelo y colaboradores fue continuado por un colaborador de Wettstein, Ricardo Benavente (1954), primero en Montevideo y ahora en Würzburg, Alemania (Benavente y Wettstein, 1977; Spindler et al., 2019). Otras contribuciones notables de este grupo son: estudios sobre la regeneración de axones (Estable et al., 1957); análisis ultraestructurales del desarrollo embrionario de pollo, incluida la primera descripción de la presencia y organización de cilias primarias en el neuroepitelio de vertebrados (Sotelo y Trujillo-Cenóz, 1958); la inervación y sinaptogénesis del órgano acústico en embriones de pollo (Vázquez-Nin y Sotelo, 1968); la ultraestructura del disco imaginal del ojo y la retina en diferenciación de la mosca (Melamed y Trujillo-Cenóz, 1975; Trujillo-Cenóz y Melamed, 1978).

Otro desarrollo técnico, que finalmente llegó a principios de la década de 1950, fue el cultivo de células y tejidos. En 1950, el entonces catedrático de Histología y Embriología de la Facultad de Medicina, Washington Buño (1909-1990), invitó al célebre histólogo y citólogo italiano Giuseppe Levi (1872-1965), quien entre otras cosas ayudó a Buño y a su colaborador Horacio Goyena a instalar el primer Laboratorio de Cultivo de Tejidos del Uruguay (Fig. 1C-E). Goyena dirigió este laboratorio durante casi 25 años, y allí formó a jóvenes investigadores en esta técnica, que él utilizó para investigar el efecto de la cortisona en el crecimiento óseo in vitro, aislando huesos largos de embriones de pollo (Buño y Goyena, 1955; Calcagno et al. al., 1970). Es importante recordar aquí que el laboratorio de Levi en Turín fue la cuna de tres futuros premios Nobel: Salvatore Luria, Renato Dulbecco y Rita Levi-Montalcini. En el mismo Departamento de Histología y Embriología, María Antonieta Rebollo (1923-2021) realizó una investigación sobre diferentes aspectos anatómicos e histoquímicos del desarrollo de los pollos, principalmente en el sistema neuromuscular (ver por ejemplo De Anda y Rebollo, 1968). Otras líneas de investigación que se iniciaron allí incluyen estudios sobre teratología 
experimental, utilizando también embriones de pollo (Castellano et al., 1973), sobre espermatogénesis de mamíferos (Micucci et al., 1971) y sobre desarrollo y ovulación de ovarios de mamíferos (Domínguez et al., 1968). Esta última línea, liderada por Roberto Domínguez Casalá, ayudó a capacitar a varios investigadores jóvenes y continúa hasta hoy en la Universidad Autónoma de México (ver una revisión reciente en Silva y Domínguez, 2020). Un lugar estrechamente relacionado donde se realizaba la investigación del desarrollo celular en esos años era la Facultad de Humanidades y Ciencias; de la cual se separó la actual Facultad de Ciencias en 1990. Allí, y desde la década de 1950, Gabriel Gerard (1919-2000) fue profesor del Departamento de Anatomía y Citología Microscópica (ahora Biología Celular). Gerard fue esencialmente un biólogo celular, cuya investigación se centró en las características histoquímicas de los gametos y las células musculares (Castellano et al., 1969).

En el área de la fisiología, es de destacar el estudio realizado por uno de nuestros neurocientíficos más reconocidos internacionalmente, Elio García-Austt (19192005), sobre el origen y desarrollo de la actividad eléctrica del cerebro del embrión de pollo (García-Austt, 1954). Otro fisiólogo de renombre, Roberto CaldeyroBarcia (1921-1996), tomó un camino diferente y en una colaboración multidisciplinar (que en algún momento incluyó a bioquímicos y electrofisiólogos, como García-Austt, pero también de manera muy importante, a médicos clínicos), sentó las bases fundamentales sobre perinatología humana utilizadas hoy en día en todo el mundo. Por sus contribuciones, Caldeyro-barcia mereció estar en la lista de candidatos al Premio Nobel en tres ocasiones (Fig. 1F; ver un ejemplo de sus muchas publicaciones en Caldeyro - Barcia y Poseiro, 1959, y una biografía completa en Beretta Curi, 2006).

\section{La diáspora y el regreso a la democracia: después de la pupa, una brillante mariposa emerge}

Desde principios de la década de 1970, en particular desde el golpe de estado de 1973, hasta 1985, el país estuvo bajo una dictadura que dio lugar a una diáspora con la mayoría de los investigadores que trabajaban en embriología, así como otras disciplinas afines. Muchos de ellos terminaron viviendo en Francia, España, Alemania, Suecia, México y otros países, donde pudieron seguir sus estudios y/o establecerse en laboratorios interesados en temas similares. Uruguay volvió a la democracia en 1985, y un año después se hizo realidad una idea revolucionaria nacida de investigadores residentes en el país y en el exterior: el PEDECIBA (Programa para el Desarrollo de las Ciencias Básicas). Este programa transversal abarca todas las instituciones académicas donde se realiza investigación científica, y tiene como principal objetivo la formación de posgrados en investigación científica. A lo largo de los primeros 20 años se logró recuperar a más de un centenar de investigadores uruguayos, ya sea residentes en el exterior o en Uruguay pero alejados de los laboratorios. Entre estos investigadores, y los cursos que organizaron, la biología del desarrollo estuvo presente por primera vez con este nombre.

El primer curso regular de Biología del Desarrollo, que aún se encuentra en marcha, fue organizado por Cristina Arruti (1944-2018), ex colaboradora de Horacio Goyena. Después de breves pasajes por los laboratorios de los famosos biólogos del desarrollo Étienne Wolff y Louis Gallien a principios de la década de 1970, Arruti regresó a París para trabajar en el desarrollo ocular en el laboratorio de Yves Courtois, donde obtuvo su doctorado. Allí descubrió un factor secretado por la retina capaz de afectar el estado de diferenciación de las células del cristalino, que resultó ser el "factor de crecimiento fibroblástico básico", o FGF2 (Arruti y Courtois, 1978). A su regreso a Uruguay en la década de 1980, Arruti rescató y dirigió el Laboratorio de Cultivo de Tejidos de Goyena, primero en la Facultad de Medicina y luego estableciéndose en la Facultad de Ciencias desde 1999 (donde fue profesora de Biología Celular hasta su jubilación en 2015). Durante este tiempo, supervisó a muchos investigadores jóvenes que aún hoy trabajan en diferentes aspectos del desarrollo del ojo y del sistema nervioso, en Uruguay y en el extranjero (ver por ejemplo Cirillo et al., 1990; De María y Arruti, 1995; Zolessi y Arruti, 2001). Un hito en la enseñanza de la biología del desarrollo fue la invitación que hizo a Claudio D. Stern (1954), otro biólogo del desarrollo nacido en Uruguay y muy influyente hoy (actualmente en el Reino Unido), quien en 1996 dio un curso experimental altamente motivador en Montevideo.

Ricardo Ehrlich (1948) también regresó en ese momento de un exilio en Francia, donde obtuvo un Doctorado en Ciencias Físicas en la Universidad Louis Pasteur, Estrasburgo. Mientras preparaba su regreso, su interacción e intercambio con otros investigadores uruguayos en Francia, entre ellos Arruti, fue un importante estímulo para que iniciara una línea de investigación sobre las bases moleculares del desarrollo en gusanos cestodos. Este tema le permitió vincular sus intereses sobre biología molecular básica con temas de interés para el país, como la lucha contra la hida- 
tidosis. Esto sucedió poco tiempo después del descubrimiento de la "homeobox" en 1983, y en medio de toda una revolución causada por la genética molecular de la mosca Drosophila en la biología del desarrollo. En ese momento, los estudios moleculares sobre gusanos planos se dirigían a un organismo modelo altamente atractivo, la planaria, pero Ehrlich no dudó en dirigir su mirada a los platelmintos parásitos. El estudio de las bases moleculares del desarrollo de Echinococcus granulosus permitió la formación de un número importante de investigadores, algunos de los cuales continúan trabajando en la biología del desarrollo de los parásitos, y que también formaron nuevas generaciones que han contribuido al conocimiento de temas fundamentales, como la determinación del eje anteroposterior en estas especies (ver por ejemplo Esteves et al., 1993; Martínez et al., 1997). Ehrlich también inició los primeros cursos de pregrado y posgrado de biología molecular en Uruguay. Los primeros alumnos de estos cursos se encuentran ahora trabajando como profesores o investigadores en todo el país. Además, promovió la estancia temporal y la finalización de la tesis doctoral de Guillermo Oliver (ahora en EE. UU.), la cual co-supervisó con E. M. De Robertis (Oliver et al., 1992). El paso de Oliver por el país influyó fuertemente en los jóvenes del laboratorio, despertando su interés por un tema recién nacido por entonces, la evo-devo (conjunción de estudios en evolución y desarrollo).

Además del establecimiento del PEDECIBA, el retorno a la democracia brindó a la Universidad la oportunidad de crear un ambicioso programa para el desarrollo de la investigación, la CSIC (Comisión Sectorial de Investigación Científica), que entre muchas otras cosas aportó fondos para becas de investigación. El gobierno también apoyó modestamente la investigación a través de diferentes programas a lo largo de este tiempo, hasta la creación en 2006 de la Agencia Nacional de Investigación e Innovación (ANII), lo que permitió una inversión mucho mayor en proyectos de investigación y becas de posgrado. El mismo año fue testigo de otro gran avance, la fundación del Institut Pasteur Montevideo; académicamente vinculado a su homónimo en París y financiado por el gobierno uruguayo, se basa en plataformas abiertas equipadas con tecnología moderna y de alto costo, algunas de las cuales no estaban disponibles antes.

Es necesario resaltar la influencia de los biólogos del desarrollo de los países vecinos en el desarrollo inicial, y especialmente reciente, de la disciplina en Uruguay. Desde el retorno a la democracia se reavivó una fuerte voluntad de colaboración científica en los países del
Cono Sur, y en particular para la Biología del Desarrollo en Uruguay, hubo una gran influencia de investigadores argentinos y chilenos. Como ejemplo, destacamos la pionera organización de un curso regional por Roberto Mayor (ahora en University College London) y Miguel Allende, en Santiago de Chile en enero de 1999. Ese curso, repetido cada dos años después, fue tremendamente influyente para todos los participantes, hasta el punto que puede ser reconocido como la semilla de la actual Sociedad Latinoamericana de Biología del Desarrollo (LASDB), fundada en 2003. En 2012, la reunión de LASDB se llevó a cabo en Montevideo, precedida por un impresionante curso de entrenamiento internacional, centrado en evo-devo (Fig. 2). Estos cursos, LASDB y los contactos que generaron, sin duda han fomentado la disciplina en Uruguay, como en otros países de la región. Además de este, otros varios eventos internacionales más específicos, aunque relacionados con el desarrollo, han tenido lugar en Uruguay en los últimos años: una serie de Simposios sobre Desarrollo Neural y Plasticidad (2008, 2012); el Primer Encuentro de la Red Latinoamericana de Pez Cebra (2010); dos simposios sobre peces anuales (2010, 2015); y el Primer Encuentro Latinoamericano de Gusanos (2017).

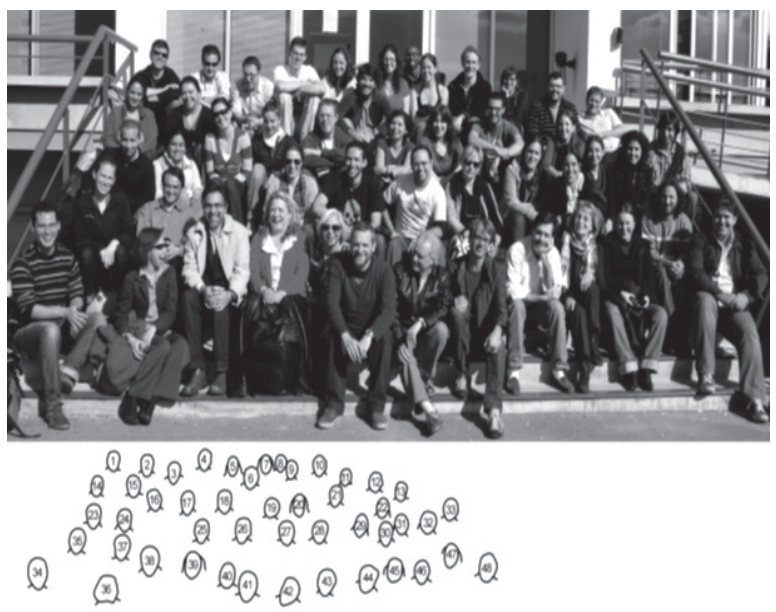

Figura 2. El curso internacional LASDB-SDB en 2012: un enfoque de biología de sistemas para comprender los mecanismos de evolución de los organismos. Todos los estudiantes y la mayor parte de los instructores, sentados en las escaleras de entrada del Institut Pasteur Montevideo. Por razones de espacio, nombramos aquí solo a los docentes y colaboradores; entre paréntesis, país de los instructores del exterior: 21, Gonzalo Aparicio; 22, Heather Marlow (Alemania); 23, Daniel Prieto; 28, Brigitte Galliot (Suiza); 29, Soledad Astrada; 30, Andrea Toledo; 34, Nicolás Papa; 36, Katia Del Rio-Tsonis (Estados Unidos); 37, Nipam Patel (Estados Unidos); 38, Alejandro Sánchez-Alvarado (Estados Unidos); 39, Billie Swalla (Estados Unidos); 40, Ida Chow (Estados Unidos); 41, Flavio Zolessi; 42, Panagiotis Tsonis (Estados Unidos); 43; Detlev 
Arendt (Alemania); 44, Rolando Rivera-Pomar (Argentina); 45; Nibia Berois; 46, María José Arezo. Instructores que no aparecen en la foto: Luis Acerenza; Siobhan Brady (Estados Unidos); María E. Castelló; Estela Castillo; Yolanda P. Cruz (Estados Unidos); Walter J. Gehring (Suiza); Enrique P. Lessa; Claudio D. Stern (Reino Unido); Andrea Streit (Reino Unido).

\section{Un presente mirando al futuro: emprendiendo el vuelo}

Como consecuencia de la creación de las agencias académicas y de financiamiento antes mencionadas y el ingreso progresivo de la comunidad científica local a la red global, incluyendo colaboraciones internacionales y formación de jóvenes investigadores, en la actualidad existen varios grupos de investigación que trabajan en diferentes áreas de la biología del desarrollo, distribuidos entre varias instituciones. Además del trabajo en organismos modelo "clásicos", como roedores, pollo, pez cebra, Drosophila melanogaster, Caenorhabditis elegans y Arabidopsis thaliana, muchos de ellos incorporados solo en los últimos 10 a 15 años, la biología del desarrollo local ha mostrado una fortaleza especial en especies no tan tradicionales, pero igualmente, o incluso más importantes. Entre ellas se encuentran los gusanos parásitos ya mencionados, y también peces autóctonos, como los peces eléctricos y los peces anuales. Como ejemplos de esto, mencionamos a continuación algunas contribuciones recientes de diferentes grupos actualmente activos.

En el amplio campo del desarrollo neural, encontramos investigaciones en vertebrados, como la neurogénesis y regeneración de la médula espinal en tortugas y ratas (Fabbiani et al., 2018; Fabbiani et al., 2020), neurogénesis y desarrollo tardío de los sistemas olfatorio y visual en peces anuales (Torres-Pérez et al., 2017), neurogénesis en el pez eléctrico Gymnotus omarorum (Olivera-Pasilio et al., 2017), plasticidad en la inervación del útero de rata (Brauer, 2016; Richeri et al., 2020), plasticidad del desarrollo de la vía visual en ratón (Vierci et al., 2016), el papel de los genes de respuesta al estrés oxidativo en el desarrollo neuronal en rata y pez cebra (López et al., 2018), el papel de la polaridad celular en la neurulación y en la diferenciación retiniana en pollo y pez cebra (Aparicio et al., 2018; Lepanto et al., 2016), y sinaptogénesis y otros aspectos del desarrollo neuronal en Drosophila (Cantera et al., 2014; Pazos Obregón et al., 2019). En los aspectos reproductivos del desarrollo, otros grupos están trabajando en la espermatogénesis y la meiosis de mamíferos desde un punto de vista molecular (Trovero et al., 2020), la diferenciación sexual y el desarrollo gonadal en peces (Vizziano-Cantonnet et al., 2016) y la regulación neuroendócrina de la inervación ovárica en ratas (Chávez-Genaro y Anesetti, 2018). Sobre diferentes aspectos de la biología molecular del desarrollo en invertebrados, destacamos la ya mencionada investigación continua sobre gusanos parásitos como los cestodos (Chalar et al., 2016; Montagne et al., 2019; Preza et al., 2018), y Drosophila (Bolatto et al., 2015). Los peces anuales son un caso particular, donde varios grupos de la Facultad de Ciencias están contribuyendo a comprender las etapas singulares del desarrollo, la regulación molecular de las diapausas, la genómica funcional de las etapas tempranas y la determinación sexual de miembros del género Austrolebias (Arezo et al., 2014; Arezo et al., 2017). En asociación con el Centro de Regulación del Genoma (Universidad de Chile), actualmente están terminando la secuenciación y ensamblaje del genoma y transcriptoma básicos de una de estas especies, A. charrua.

\section{Observaciones finales}

Hemos intentado aquí una descripción resumida del advenimiento "no canónico" de la biología del desarrollo en Uruguay, no directamente desde la embriología, sino desde otras disciplinas como la citología y la fisiología, reforzadas posteriormente por el regreso de investigadores del exilio. Las escuelas que iniciaron, sumadas a la interacción con biólogos del desarrollo regionales e internacionales, llevaron a los diversos grupos de investigación que encontramos hoy. A medida que estos grupos crezcan y los estudiantes se gradúen o regresen de su formación en el extranjero, en los próximos años se espera una diversificación sostenida y un fortalecimiento de la biología del desarrollo uruguaya.

\section{Agradecimientos}

Los autores agradecen a las siguientes personas su ayuda con comentarios, relatos y materiales que fueron fundamentales para la redacción de este manuscrito: Carmen Álvarez, Gabriel Anesetti, Ricardo Benavente, Miguel Castellano, Rebeca Chávez, Adriana Geisinger, Susana González, Mario Lalinde, Eduardo López, Milka Radmilovich, Gabriel Santoro, Ángela Santurio, José R. Sotelo-Silveira, Omar Trujillo-Cenóz, Pablo Uriarte. 


\section{Referencias}

Aparicio, G., Arruti, C., \& Zolessi, F. R. (2018). MARCKS phosphorylation by PKC strongly impairs cell polarity in the chick neural plate. Genesis, 56(4), e23104. https://doi.org/10.1002/dvg.23104

Arezo, M. J., Papa, N. G., Berois, N., Clivio, G., Montagne, J., \& De la Piedra, S. (2017). Annual killifish adaptations to ephemeral environments: Diapause I in two Austrolebias species. Developmental Dynamics, 246(11), 848-857. https://doi.org/10.1002/dvdy.24580

Arezo, M. J., Papa, N., Guttierrez, V., García, G., \& Berois, N. (2014). Sex determination in annual fishes: Searching for the master sex-determining gene in Austrolebias charrua (Cyprinodontiformes, Rivulidae). Genetics and Molecular Biology, 37(2), 364-374. https://doi.org/10.1590/S141547572014005000009

Arruti, C., \& Courtois, Y. (1978). Morphological changes and growth stimulation of Bovine Epithelial Lens cells by a retinal extract in vitro. Experimental Cell Research, 117(2), 283-292. https://doi. org/10.1016/0014-4827(78)90142-8

Benavente, R., \& Wettstein, R. (1977). An ultrastructural cytogenetic study on the evolution of sex chromosomes during the spermatogenesis of Lycosa malitiosa (Arachnida). Chromosoma, 64(3), 255-277. https://doi.org/10.1007/BF00328081

Beretta Curi, A. (2006). Roberto Caldeyro Barcia: el mandato de una vocación. PEDECIBA/Ediciones Trilce.

Bolatto, C., Parada, C., Revello, F., Zuñiga, A., Cabrera, P., \& Cambiazo, V. (2015). Spatial and temporal distribution of Patched-related protein in the Drosophila embryo. Gene Expression Patterns, 19(1-2), 120-128. https://doi.org/10.1016/j. gep.2015.10.002

Brauer, M. (2016). Plasticity in Uterine Innervation: State of the Art. Current Protein \& Peptide Science, 18(2), 108-119. https://doi.org/10.2174/1389203 717666160322145411

Buño, W., \& Goyena, H. (1955). Effect of cortisone upon growth in vitro of femur of the chick embryo. Proceedings of the Society for Experimental Biology and Medicine, 89(4), 622-625. https://doi. org/10.3181/00379727-89-21896

Calcagno, M., Goyena, H., Arrambide, E., \& Arruti de Urse, C. (1970). Action of cortisone and cortisol upon biosynthesis of chondroitin sulfate in femur in vitro cultures of chick embryo. Experimental Cell Research, 63(1), 131-137. https://doi. org/10.1016/0014-4827(70)90340-X

Caldeyro-Barcia, R., \& Poseiro, J. J. (1959). Oxytocin and contractility of the pregnant human uterus. Annals of the New York Academy of Sciences, 75(2), 813830. https://doi.org/10.1111/j.1749-6632.1959. tb44593.x

Campos Muñoz, A. (2013). Eduardo de Robertis. En el centenario de su nacimiento (1913-2013). Actualidad Médica, 790, 167-170. https:// actualidadmedica.es/articulo/790_hca01

Cantera, R., Ferreiro, M. J., Aransay, A. M., \& Barrio, R. (2014). Global gene expression shift during the transition from early neural development to late neuronal differentiation in Drosophila melanogaster. PLoS ONE, 9(5), e97703. https:// doi.org/10.1371/journal.pone.0097703

Castellano, M. A., Tórtora, J. L., Germino, N. I., Rama, F., \& Ohanian, C. (1973). The effects of isonicotinic acid hydrazide on the early chick embryo. Journal of Embryology and Experimental Morphology, 29(1), 209-219. https://doi.org/10.1242/ dev.29.1.209

Castellano, Miguel A., Germino, N. I., Berois de Haro, N., \& Gerard, G. (1969). Histochemical demonstration of I-amino acid-tetrazolium reductase. Histochemie, 18(3), 277-280. https:// doi.org/10.1007/BF00306174

Chalar, C., Martínez, C., Brauer, M. M., Ehrlich, R., \& Marín, M. (2016). Eghbx2, a homeobox gene involved in the maturation of calcified structures in Echinococcus granulosus. Gene Reports, 3, 3946. https://doi.org/10.1016/j.genrep.2016.02.001

Chávez-Genaro, R., \& Anesetti, G. (2018). First ovarian response to gonadotrophin stimulation in rats exposed to neonatal androgen excess. Journal of Molecular Histology, 49(6), 631-637. https://doi. org/10.1007/s10735-018-9800-5

Cirillo, A., Arruti, C., Courtois, Y., \& Jeanny, J. C. (1990). Localization of basic fibroblast growth factor binding sites in the chick embryonic neural retina. Differentiation, 45(3), 161-167. https://doi. org/10.1111/j.1432-0436.1990.tb00469.x

De Anda, G., \& Rebollo, M. A. (1968). Histochemistry of the neuromuscular spindles in the chicken during development. Acta Histochemica, 31(2), 287-295. 
de Maria, A., \& Arruti, C. (1995). a-crystallin polypeptides in developing chicken lens cells. Experimental Eye Research, 61(2), 181-187. https://doi.org/10.1016/S0014-4835(05)80038-7

De Robertis, E. (1956). Morphogenesis of the retinal rods; an electron microscope study. The Journal of Biophysical and Biochemical Cytology, 2(4, Suppl), 209-218. https://doi.org/10.1083/jcb.2.4.209

Domínguez, R., Carlevaro, E., \& Buño, W. (1968). Evolution of ovarian grafts in male guinea-pigs castrated the first day of life. Experientia, 24(5), 459-460. https://doi.org/10.1007/BF02144390

Drets, M. E. (2013). Francisco Alberto Sáez - Primer citogenetista de América Latina. DIRAC, Facultad de Ciencias.

Estable, C., Acosta-Ferreira, W., \& Sotelo, J. R. (1957). An electron microscope study of the regenerating nerve fibers. Zeitschrift Für Zellforschung Und Mikroskopische Anatomie, 46(4), 387-399. https://doi.org/10.1007/BF00345052

Esteves, A., Dallagiovanna, B., \& Ehrlich, R. (1993). A developmentally regulated gene of Echinococcus granulosus codes for a 15.5-kilodalton polypeptide related to fatty acid binding proteins. Molecular and Biochemical Parasitology, 58(2), 215-222. https://doi.org/10.1016/0166-6851(93)90043-W

Fabbiani, G., Reali, C., Valentín-Kahan, A., Rehermann, M. I., Fagetti, J., Falco, M. V., \& Russo, R. E. (2020). Connexin signaling is involved in the reactivation of a latent stem cell niche after spinal cord injury. Journal of Neuroscience, 40(10), 2246-2258. https://doi.org/10.1523/ JNEUROSCI.2056-19.2020

Fabbiani, G., Rehermann, M. I., Aldecosea, C., TrujilloCenóz, O., \& Russo, R. E. (2018). Emergence of serotonergic neurons after spinal cord injury in turtles. Frontiers in Neural Circuits, 12, 20. https:// doi.org/10.3389/fncir.2018.00020

Garcia-Austt, E. (1954). Development of electrical activity in cerebral hemispheres of the chick embryo. Proceedings of the Society for Experimental Biology and Medicine, 86(2), 348352. https://doi.org/10.3181/00379727-86-21095

Lepanto, P., Davison, C., Casanova, G., Badano, J. L., \& Zolessi, F. R. (2016). Characterization of primary cilia during the differentiation of retinal ganglion cells in the zebrafish. Neural Development, 11(1), 10. https://doi.org/10.1186/s13064-016-0064-z
López, L., Zuluaga, M. J., Lagos, P., Agrati, D., \& Bedó, G. (2018). The expression of Hypoxia-Induced Gene 1 (Higd1a) in the central nervous system of male and female rats differs according to age. Journal of Molecular Neuroscience, 66(3), 462-473. https:// doi.org/10.1007/s12031-018-1195-y

Martínez, C., Chalar, C., González, J., \& Ehrlich, R. (1997). The homeobox-containing gene EgHbx3 from Echinococcus granulosus is expressed in the stalk of protoscoleces. International Journal for Parasitology, 27(11), 1379-1381. https://doi. org/10.1016/S0020-7519(97)00082-9

Melamed, J., \& Trujillo-Cenóz, O. (1975). The fine structure of the eye imaginal disks in muscoid flies. Journal of Ultrastructure Research, 51(1), 79-93. https://doi.org/10.1016/S0022-5320(75)80010-4

Micucci, M., Rama, F., Castellano, M. A., \& Germino, N. I. (1971). The histochemical distribution of fructose metabolism enzymes in bovine spermatogenesis. Journal of Anatomy, 109(2), 209-214. https://www.ncbi.nlm.nih.gov/pmc/ articles/PMC1271001

Montagne, J., Preza, M., Castillo, E., Brehm, K., \& Koziol, U. (2019). Divergent Axin and GSK-3 paralogs in the beta-catenin destruction complexes of tapeworms. Development Genes and Evolution, 229(4), 89-102. https://doi.org/10.1007/s00427019-00632-w

Oliver, G., Vispo, M., Mailhos, A., Martínez, C., SosaPineda, B., Fielitz, W., \& Ehrlich, R. (1992). Homeoboxes in flatworms. Gene, 121(2), 337-342. https://doi.org/10.1016/0378-1119(92)90140-K

Olivera-Pasilio, V., Lasserre, M., \& Castelló, M. E. (2017). Cell proliferation, migration, and neurogenesis in the adult brain of the pulse type weakly electric fish, Gymnotus omarorum. Frontiers in Neuroscience, 11(AUG), 437. https:// doi.org/10.3389/fnins.2017.00437

Pazos Obregón, F., Palazzo, M., Soto, P., Guerberoff, G., Yankilevich, P., \& Cantera, R. (2019). An improved catalogue of putative synaptic genes defined exclusively by temporal transcription profiles through an ensemble machine learning approach. BMC Genomics, 20(1), 1011. https:// doi.org/10.1186/s12864-019-6380-z

Pellegrino, V., Klimavicius, S., Vique, M. I., \& Varela, G. (Eds.). (2017). Científicos uruguayos: destacadas contribuciones al conocimiento biológico durante 
los siglos XIX y XX. Grupo Magro Editores. http:// repositorio.cfe.edu.uy/handle/123456789/993

Preza, M., Montagne, J., Costábile, A., Iriarte, A., Castillo, E., \& Koziol, U. (2018). Analysis of classical neurotransmitter markers in tapeworms: Evidence for extensive loss of neurotransmitter pathways. International Journal for Parasitology, 48(13), 979992. https://doi.org/10.1016/j.ijpara.2018.06.004

Richeri, A., Vierci, G., Martínez, G. F., Latorre, M. P., Chalar, C., \& Brauer, M. M. (2020). Neuropilin-1 receptor in the rapid and selective estrogeninduced neurovascular remodeling of rat uterus. Cell and Tissue Research, 381(2), 299-308. https:// doi.org/10.1007/s00441-020-03196-8

Silva, C. C., \& Domínguez, R. (2020). Clock control of mammalian reproductive cycles: Looking beyond the pre-ovulatory surge of gonadotropins. In Reviews in Endocrine and Metabolic Disorders (Vol. 21, Issue 1, pp. 149-163). Springer. https:// doi.org/10.1007/s11154-019-09525-9

Sotelo, J. R. (1959). An electron microscope study on the cytoplasmic and nuclear components of rat primary oocytes. Zeitschrift Für Zellforschung Und Mikroskopische Anatomie, 50(6), 749-765. https://doi.org/10.1007/BF00342364

Sotelo, J. R., \& Trujillo-Cenóz, O. (1958). Electron microscope study on the development of ciliary components of the neural epithelium of the chick embryo. Zeitschrift Für Zellforschung Und Mikroskopische Anatomie, 49(1), 1-12. https:// doi.org/10.1007/BF00335059

Sotelo, J. R., \& Trujillo-Cenóz, O. (1960). Electron microscope study on spermatogenesis Chromosome morphogenesis at the onset of meiosis (cyte I) and nuclear structure of early and late spermatids. Zeitschrift Für Zellforschung Und Mikroskopische Anatomie, 51(3), 243-277. https://doi.org/10.1007/BF00339968

Sotelo, J. R., \& Wettstein, R. (1964). Electron microscope study on meiosis - The sex chromosome in spermatocytes, spermatids and oocytes of Gryllus argentinus. Chromosoma, 15(4), 389-415. https:// doi.org/10.1007/BF00368139

Spindler, M. C., Filbeck, S., Stigloher, C., \& Benavente, R. (2019). Quantitative basis of meiotic chromosome synapsis analyzed by electron tomography. Scientific Reports, 9(1), 16102. https://doi. org/10.1038/s41598-019-52455-4
Torres-Pérez, M., Rosillo, J. C., Berrosteguieta, I., Olivera-Bravo, S., Casanova, G., García-Verdugo, J. M., \& Fernández, A. S. (2017). Stem cells distribution, cellular proliferation and migration in the adult Austrolebias charrua brain. Brain Research, 1673, 11-22. https://doi.org/10.1016/j. brainres.2017.08.003

Trovero, M. F., Rodríguez-Casuriaga, R., Romeo, C., Santiñaque, F. F., François, M., Folle, G. A., Benavente, R., Sotelo-Silveira, J. R., \& Geisinger, A. (2020). Revealing stage-specific expression patterns of long noncoding RNAs along mouse spermatogenesis. RNA Biology, 17(3), 350-365. https://doi.org/10.1080/15476286.2019.1700332

Trujillo-Cenóz, O., \& Melamed, J. (1978). Development of photoreceptor patterns in the compound eyes of muscoid flies. Journal of Ultrastructure Research, 64(1), 46-62. https://doi.org/10.1016/ S0022-5320(78)90006-0

Vázquez-Nin, G. H., \& Sotelo, J. R. (1968). Electron microscope study of the developing nerve terminals in the acoustic organs of the chick embryo. Zeitschrift Für Zellforschung Und Mikroskopische Anatomie, 92(3), 325-338. https://doi.org/10.1007/BF00455590

Vierci, G., Pannunzio, B., Bornia, N., \& Rossi, F. M. (2016). H3 and H4 lysine acetylation correlates with developmental and experimentally induced adult experience-dependent plasticity in the mouse visual cortex. Journal of Experimental Neuroscience, 2016, 49-64. https://doi. org/10.4137/JEN.S39888

Vizziano-Cantonnet, D., Di Landro, S., Lasalle, A., Martínez, A., Mazzoni, T. S., \& Quagio-Grassiotto, I. (2016). Identification of the molecular sexdifferentiation period in the siberian sturgeon. Molecular Reproduction and Development, 83(1), 19-36. https://doi.org/10.1002/mrd.22589

Wettstein, R., \& Sotelo, J. R. (1967). Electron microscope serial reconstruction of spermatocyte nuclei at pachytene. J. Microscopie, 6, 557-576.

Zolessi, F. R., \& Arruti, C. (2001). Sustained phosphorylation of MARCKS in differentiating neurogenic regions during chick embryo development. Brain Research. Developmental Brain Research, 130(2), 257-267. https://doi. org/10.1016/S0165-3806(01)00251-6 